\title{
Minimum-error discrimination between two sets of similarity-transformed quantum states
}

\author{
M. A. Jafarizadeh, ${ }^{1,2,3,}{ }^{*}$ Y. Mazhari Khiavi, ${ }^{1,}{ }^{\dagger}$ Y. Akbari Kourbolagh, ${ }^{4, \ddagger}$ \\ ${ }^{1}$ Department of Theoretical Physics and Astrophysics, University of Tabriz, Tabriz 51664, Iran. \\ 2 Institute for Studies in Theoretical Physics and Mathematics, Tehran 19395-1795, Iran. \\ ${ }^{3}$ Research Institute for Fundamental Sciences, Tabriz 51664, Iran. \\ ${ }^{4}$ Department of Physics, Azarbaijan University of Tarbiat Moallem, 53714-161 Tabriz, Iran.
}

January 23, 2021

\begin{abstract}
Using the new form of necessary and sufficient conditions introduced in Ref. [16, minimum error discrimination among two sets of similarity transformed equiprobable quantum qudit states is investigated. In the case that the unitary operators are generating sets of two irreducible representations, the optimal set of measurements and the corresponding maximum success probability of discrimination are determined in closed form. In the case of reducible representations, there exists no closed-form formula in general, but the procedure can be applied in each case accordingly. Finally, we give the maximum success probability of optimal discrimination for some important examples of mixed quantum states, such as qubit states together with three special cases and generalized Bloch sphere m-qubit states.

Keywords: Minimum error discrimination, Similarity transformed quantum states, Probability operator measure

PACs Index: 01.55.+b, 02.10.Yn
\end{abstract}

\section{Introduction}

The discrimination of nonorthogonal quantum states is an important and challenging problem in the realm of quantum information theory. It is known that one cannot discriminate perfectly among nonorthogonal quantum states and ought to invoke to an optimal way of state discrimination. There exist two approaches to the problem of discrimination: optimal minimum-error discrimination in which the state identification is probabilistic [1]-[8] and optimal unambiguous discrimination in which the states are discriminated without error [9][14]. Here, we consider discrimination strategies, known as the minimum-error discrimination

\footnotetext{
*E-mail:jafarizadeh@tabrizu.ac.ir

$\dagger^{\dagger}$ E-mail:mazhari@tabrizu.ac.ir

${ }_{\ddagger}^{\ddagger}$ E-mail:yakbari@azaruniv.edu
} 
(MED), which are based upon the minimization of the rate error. For treating minimum error discrimination, there exist two known strategies : the necessary and sufficient conditions for optimal discrimination [1]-5] and Helstrom family of ensembles [15]. However, solving problems by means of them, except for some particular cases, is a difficult task. In Ref. [16], a new technique has been presented in which the two previous strategies are comprised to obtain optimality conditions of the equality form and it has been shown that the new technique is powerful in solving problems of optimal discrimination between mixed quantum states which are in general not symmetric. Here, we use the latter technique in the problem of minimum error discrimination among two different sets of equiprobable similarity transformed quantum states.

In this paper, we first use the technique of Ref. [16] to investigate MED between two sets of equiprobable quantum mixed states generated from two original density operators by unitary similarity transformations. The novelty of this work is that quantum states to be discriminated are partitioned into two sets with non-equal probabilities. In the case that the unitary operators are irreducible representations of generators of a subgroup of unitary group $U(d)$, the maximum success probability and optimal measurement operators are precisely derived. In the case that the unitary operators are reducible representations of the corresponding subgroup, although there exists in general no closed-form formula but the procedure can be applied in each case accordingly. Finally, we study MED between some important classes of mixed quantum states such as generalized Bloch sphere m-qubit states, in details.

\section{Minimum error discrimination among states of two sets of similarity transformed equiprobable states}

In general, the measurement strategy is described in terms of a set of positive semidefinite operators $\Pi_{i}$ known as the probability operator measure (POM). The measurement outcome labeled by $i$ is associated with the operator $\Pi_{i}$ and the sum of the POM elements must be the identity operator, i.e., $\sum_{i} \Pi_{i}=\mathrm{I}$ which is known as a resolution of the identity. Knowing that the transmitted state is $\rho_{j}$, the probability of observing the outcome $i$ by the receiver is $p(i \mid j)=\operatorname{Tr}\left(\Pi_{i} \rho_{j}\right)$. Let us consider the different states $\rho_{1}, \rho_{2}, \ldots, \rho_{N}$ with prior probabilities $p_{1}, p_{2}, \ldots, p_{N}$, respectively $\left(p_{i} \geq 0, \sum_{i} p_{i}=1\right)$. Then the success probability $p$ for correctly identifying the states $\rho_{i}$ is given by

$$
p=\sum_{i=1}^{N} p_{i} \operatorname{Tr}\left(\rho_{i} \Pi_{i}\right) .
$$

The necessary and sufficient conditions leading to the minimum-error discrimination can be written as [16]

$$
\mathcal{M}=p_{j} \rho_{j}+\left(p_{\text {opt }}-p_{j}\right) \tau_{j}, \quad j=1, \ldots, N .
$$

in which $\mathcal{M}$ denotes $\sum_{i=1}^{N} p_{i} \Pi_{i} \rho_{i}, p_{\text {opt }}$ stands for maximal success probability, $p_{\text {opt }} \geq p_{j}$ and $\tau_{j}$ 's are positive operators of trace one called conjugate states [15]. If $\Pi_{k}=I$ for some $k \in\{1, \ldots N\}$, then taking trace of both sides of Eq. (2) gives $p_{\text {opt }}=p_{k}$. Note that for two 
different equiprobable states $\rho_{k}$ and $\rho_{l}$ it is impossible that $p_{\text {opt }}=p_{k}=p_{l}$, since in this case Eq. (2) implies $\rho_{k}=\rho_{l}$ which is a contradiction. It is shown in Ref. [16] that the conditions (2) lead to

$$
\left(p_{\text {opt }}-p_{j}\right) \operatorname{Tr}\left(\tau_{j} \Pi_{j}\right)=0, \quad j=1, \ldots, N \text {; }
$$

In this paper, we consider two sets of $N$ different states: the first set containing equiprobable unprimed states with prior probabilities $\eta$ and the second set containing equiprobable primed states with prior probabilities $\eta^{\prime}$ as follows

$$
\begin{gathered}
\rho_{j}=U_{j} \rho_{1} U_{j}^{-1}, \quad \tau_{j}=U_{j} \tau_{1} U_{j}^{-1}, \quad j=1, \ldots, n, \\
\rho_{j}^{\prime}=U_{j}^{\prime} \rho_{1}^{\prime} U_{j}^{\prime-1}, \quad \tau_{j}^{\prime}=U_{j}^{\prime} \tau_{1}^{\prime} U_{j}^{\prime-1}, \quad j=1, \ldots, n^{\prime},
\end{gathered}
$$

where $n+n^{\prime}=N$ and $\left\{U_{1}=I_{d}, U_{2}, \ldots, U_{n}\right\}$ and $\left\{U_{1}^{\prime}=I_{d}, U_{2}^{\prime}, \ldots, U_{n^{\prime}}^{\prime}\right\}$ are generating sets of representations of two subgroups of $U(d)$, the group of all $d \times d$ unitary matrices. Clearly, we have

$$
n \eta+n^{\prime} \eta^{\prime}=1
$$

Such states is referred to as similarity transformed states. Denoting POM elements corresponding to $\rho_{j}$ and $\rho_{j}^{\prime}$ by $\Pi_{j}$ and $\Pi_{j}^{\prime}$ respectively, we must have a resolution of the identity

$$
\sum_{j=1}^{n} \Pi_{j}+\sum_{j=1}^{n^{\prime}} \Pi_{j}^{\prime}=I
$$

Assume that $\Pi_{j}=\lambda_{j} \pi_{j}$ and $\Pi_{j}^{\prime}=\lambda_{j}^{\prime} \pi_{j}^{\prime}$ for some nonnegative numbers $\lambda_{j}$ and $\lambda_{j}^{\prime}$ and positive semidefinite operators $\pi_{j}$ and $\pi_{j}^{\prime}$ such that $\sum_{j=1}^{n} \lambda_{j}+\sum_{j=1}^{n^{\prime}} \lambda_{j}^{\prime}=1$. As POM operators constitute a resolution of the identity, it is possible that for the optimal POM some of the operators $\Pi_{i}$ and $\Pi_{i}^{\prime}$ vanish. Note here that $\pi_{j}$ 's and $\pi_{j}^{\prime}$ 's must be satisfy the conditions (3)

$$
\left(p_{\text {opt }}-\eta\right) \lambda_{j} \operatorname{Tr}\left(\tau_{j} \pi_{j}\right)=\left(p_{\text {opt }}-\eta^{\prime}\right) \lambda_{j}^{\prime} \operatorname{Tr}\left(\tau_{j}^{\prime} \pi_{j}^{\prime}\right)=0
$$

so the optimal operators $\pi_{j}$ and $\pi_{j}^{\prime}$ can also be assumed to obtain from positive operators $\pi_{1}$ and $\pi_{1}^{\prime}$ respectively via the same similarity transform which defines the states $\rho_{i}$ and $\rho_{i}^{\prime}$ and the corresponding conjugate states $\tau_{i}$ and $\tau_{i}^{\prime}$, i.e.

$$
\begin{aligned}
& \pi_{i}=U_{i} \pi_{1} U_{i}^{-1}, \\
& \pi_{i}^{\prime}=U_{i} \pi_{1}^{\prime} U_{i}^{-1} .
\end{aligned}
$$

Therefore, Eq. (8) is reduced to

$$
\left(p_{\text {opt }}-\eta\right) \lambda_{j} \operatorname{Tr}\left(\tau_{1} \pi_{1}\right)=\left(p_{\text {opt }}-\eta^{\prime}\right) \lambda_{j}^{\prime} \operatorname{Tr}\left(\tau_{1}^{\prime} \pi_{1}^{\prime}\right)=0 .
$$

Here, optimality conditions (2) can clearly be written as

$$
\begin{aligned}
\mathcal{M} & =\eta \rho_{i}+\left(p_{\text {opt }}-\eta\right) \tau_{i} \\
& =\eta^{\prime} \rho_{j}^{\prime}+\left(p_{\text {opt }}-\eta^{\prime}\right) \tau_{j}^{\prime} .
\end{aligned}
$$


where $\mathcal{M}$ denotes $\eta \sum_{i=1}^{n} \Pi_{i} \rho_{i}+\eta^{\prime} \sum_{i=1}^{n^{\prime}} \Pi_{i}^{\prime} \rho_{i}^{\prime}$. From (41), (15) and (12), it is easy to see that

$$
\mathcal{M}=U_{i} \mathcal{M} U_{i}^{-1}=U_{j}^{\prime} \mathcal{M} U_{j}^{\prime-1}
$$

We know that the representations $U_{i}$ 's and $U_{j}^{\prime}$ 's are either irreducible or reducible. We discuss the two cases separately.

\subsection{The irreducible case}

Let $U_{i}$ 's and $U_{j}^{\prime}$ 's be generating sets of irreducible representations of two subgroups of $U(d)$. Then, by the Schur's first lemma on representation theory [17, 18], Eq. (13) implies that $\mathcal{M}$ is a multiple of identity operator; i.e., $\mathcal{M}=\alpha I$ for some complex number $\alpha$.

By taking trace of Eq. (12), we get

$$
\alpha=\frac{p_{o p t}}{d} .
$$

In order to obtain an optimal measurement for discrimination among states $\rho_{i}$, let us write $\rho_{1}$ in the spectral decomposition form as $\rho_{1}=\sum_{i=1}^{d} a_{i}|i\rangle\langle i|$. Then, Eq. (12) implies that $\tau_{1}$ is also diagonal in the basis $|i\rangle$, say, $\tau_{1}=\sum_{i=1}^{d} b_{i}|i\rangle\langle i|$. By the replacement of $\rho_{1}$ and $\tau_{1}$ in Eq. (12), we have

$$
\frac{p_{\text {opt }}}{d} \sum_{i=1}^{d}|i\rangle\left\langle i\left|=\eta \sum_{i=1}^{d} a_{i}\right| i\right\rangle\left\langle i\left|+\left(p_{\text {opt }}-\eta\right) \sum_{i=1}^{d} b_{i}\right| i\right\rangle\langle i|,
$$

and hence

$$
p_{\text {opt }}=d\left[\eta a_{i}+\left(p_{\text {opt }}-\eta\right) b_{i}\right]
$$

Similarly, for the second set, we have

$$
p_{o p t}=d\left[\eta^{\prime} a_{i}^{\prime}+\left(p_{o p t}-\eta^{\prime}\right) b_{i}^{\prime}\right]
$$

Since, for the irreducible case we have $n \geq 2$ and $n^{\prime} \geq 2$, then as mentioned above $p_{\text {opt }} \neq \eta, \eta^{\prime}$ and hence Eq. (8) implies that

$$
\lambda_{j} \operatorname{Tr}\left(\tau_{1} \pi_{1}\right)=\lambda_{j}^{\prime} \operatorname{Tr}\left(\tau_{1}^{\prime} \pi_{1}^{\prime}\right)=0
$$

Hence, either $\lambda_{j}=0\left(\lambda_{j}^{\prime}=0\right)$ or $\pi_{1}\left(\pi_{1}^{\prime}\right)$ is perpendicular to $\tau_{1}\left(\tau_{1}^{\prime}\right)$; namely, $\tau_{1}\left(\tau_{1}^{\prime}\right)$ is not full rank. In the latter case, Eq. (16) indicates that when eigenvalues $a_{i}$ of $\rho_{1}$ are all distinct, only one of the coefficients $b_{i}$, say $b_{l}$, is zero and $a_{l}$ is the greatest eigenvalue of $\rho_{1}$, denoted as $a_{\max }$. Thus,

$$
p_{\text {opt }}=\eta a_{\text {max }} d
$$

and $\pi_{1}=\beta_{l}|l\rangle\langle l|$ with $\beta_{l}$ an unknown positive constant and

$$
\Pi_{i}=\lambda_{i} \beta_{l} U_{i}|l\rangle\langle l| U_{i}^{-1} .
$$


Now, let there exist $m$ eigenvectors $i_{1}, \ldots, i_{m}$ of $\rho_{1}$ with the same eigenvalue $a_{\text {max }}$. Then, $p_{\text {opt }}$ is again given by (19) and by Eq. (16), these eigenvectors must correspond to the eigenvalue zero of $\tau_{1}$. So, the operator $\pi_{1}$ can be written as

$$
\pi_{1}=\alpha_{1}\left|i_{1}\right\rangle\left\langle i_{1}\left|+\ldots+\alpha_{m}\right| i_{m}\right\rangle\left\langle i_{m}\right|,
$$

where $\alpha_{i}$ 's are non-negative numbers. By the same argument, we can get for the second set relations similar to Eqs. (19), (20) and (21). If this equality do not hold, all elements of one of sets $\left\{b_{i}\right\}_{i=1}^{n}$ and $\left\{b_{i}^{\prime}\right\}_{i=1}^{n^{\prime}}$ are nonzero.

If $\tau_{1}\left(\tau_{1}^{\prime}\right)$ be full rank, then it cannot be perpendicular to $\pi_{1}\left(\pi_{1}^{\prime}\right)$ and by Eq. (18), $\lambda_{j}$ 's $\left(\lambda_{j}^{\prime}\right.$ 's) are all necessarily zero. When this is the case, operators of optimal POM corresponding to the first (second) set are all zero and the unprimed (primed) states do not enter in the discrimination.

\subsection{The reducible case}

Let $U_{i}$ 's and $U_{j}^{\prime}$ 's be generating sets of reducible representations. Then, it is shown that the invariance of $\mathcal{M}$ under the operators $U_{i}$ 's and $U_{j}^{\prime}$ 's (see Eq. (13)) requires that $\mathcal{M}$ is diagonal; i.e., $\mathcal{M}=\operatorname{diag}\left(M_{1}, \ldots, M_{d}\right)$ (for a proof, see Appendix B of [16]).

Here, the same technique as the irreducible case is applicable. However, we cannot give a general solution because the explicit form of $M$ differs per case. In what follows, we illustrate the problem by considering some examples of qubit and $m$-qubit mixed states.

\section{Examples}

\section{MED between two sets of similarity transformed equiprobable qubit states}

Let us consider two different qubit states as

$$
\begin{aligned}
& \rho_{1}=\frac{1}{2}\left(I+b \hat{n}^{(1)} \cdot \vec{\sigma}\right), \\
& \rho_{1}^{\prime}=\frac{1}{2}\left(I+b^{\prime} \hat{n}^{\prime(1)} \cdot \vec{\sigma}\right),
\end{aligned}
$$

where $\sigma_{i}$ 's are the Pauli matrices and $\hat{n}^{(1)}$ and $\hat{n}^{\prime(1)}$ are unit vectors. Furthermore, let $U_{j}$ and $U_{j}^{\prime}$ be arbitrary rotations about the z-axis that rotate $\rho_{1}$ and $\rho_{1}^{\prime}$ into $\rho_{j}$ and $\rho_{j}^{\prime}$, respectively, such that

$$
\begin{aligned}
\rho_{j} & =\frac{1}{2}\left(I+b \hat{n}^{(j)} \cdot \vec{\sigma}\right), \\
\rho_{j}^{\prime} & =\frac{1}{2}\left(I+b^{\prime} \hat{n}^{\prime(j)} \cdot \vec{\sigma}\right) .
\end{aligned}
$$

The conjugate states corresponding to $\rho_{j}$ and $\rho_{j}^{\prime}$ have the form

$$
\tau_{j}=\frac{1}{2}\left(I+c \hat{m}^{(j)} \cdot \vec{\sigma}\right)
$$




$$
\tau_{j}^{\prime}=\frac{1}{2}\left(I+c^{\prime} \hat{m}^{\prime(j)} \cdot \vec{\sigma}\right)
$$

where $\hat{m}^{(j)}$ and $\hat{m}^{\prime(j)}$ are unit vectors. Now, from the invariance of $\mathcal{M}$ under rotations about the $z$-axis, it follows that $n>1$ or $n^{\prime}>1$ and

$$
\mathcal{M}=\alpha I_{d}+\beta \sigma_{z}
$$

First, we assume that $n \geq 2$ and $n^{\prime} \geq 2$. Hence, we have $p_{o p t}>\eta, \eta^{\prime}$ and by Eq. (8), $\lambda_{j} \operatorname{Tr}\left(\tau_{j} \pi_{j}\right)=\lambda_{j}^{\prime} \operatorname{Tr}\left(\tau_{j}^{\prime} \pi_{j}^{\prime}\right)=0$. This consideration together with resolution of the identity imply that at least one of $\tau_{1}$ and $\tau_{1}^{\prime}$ is not full rank. Without loss of generality, let us assume that $\tau_{1}$ is not full rank such that its minimum eigenvalue $\frac{1-c}{2}$ is zero which yields $c=1$. To satisfy Eq. (8), the optimal POM elements must have the following expression

$$
\begin{gathered}
\Pi_{j}=\lambda_{j} U_{j} \pi_{1} U_{j}^{-1}=\lambda_{j}\left(I-m_{x}^{(j)} \sigma_{x}-m_{y}^{(j)} \sigma_{y}-m_{z} \sigma_{z}\right), \quad \lambda_{j} \geq 0, \\
\Pi_{j}^{\prime}=\lambda_{j}^{\prime} U_{j} \pi_{1}^{\prime} U_{j}^{-1}=\lambda_{j}^{\prime}\left(I-m_{x}^{\prime(j)} \sigma_{x}-m_{y}^{\prime(j)} \sigma_{y}-m_{z}^{\prime} \sigma_{z}\right), \quad \lambda_{j}^{\prime} \geq 0 .
\end{gathered}
$$

By substituting the expressions of $\Pi_{j}$ and $\Pi_{j}^{\prime}$ from Eqs. (29) and (30) into Eq. (7), it is easy to see that the following restrictions are imposed on $\lambda_{j}$ and $\lambda_{j}^{\prime}$

$$
\begin{gathered}
\sum_{j=1}^{n} \lambda_{j}+\sum_{j=1}^{n^{\prime}} \lambda_{j}^{\prime}=1 \\
m_{z} \sum_{j=1}^{n} \lambda_{j}+m_{z}^{\prime} \sum_{j=1}^{n^{\prime}} \lambda_{j}^{\prime}=0 \\
\sum_{j=1}^{n} \lambda_{j}\left(m_{x}^{(j)} \mathbf{i}+m_{y}^{(j)} \mathbf{j}\right)+\sum_{j=1}^{n^{\prime}} \lambda_{j}^{\prime}\left(m_{x}^{\prime(j)} \mathbf{i}+m_{y}^{\prime(j)} \mathbf{j}\right)=0 .
\end{gathered}
$$

It is clear from Eqs. (31) and (32) that the signs of $m_{z}$ and $m_{z}^{\prime}$ are opposite unless $m_{z}=$ $m_{z}^{\prime}=0$. Furthermore, Eqs. (32) and (33) imply that the points representing the optimal measurement operators do not share the same hemisphere of the Bloch sphere.

In what follows, it is convenient to obtain relations between components of Bloch vectors of the states and whose conjugate states. To this end, for the case $n>1$ and $n^{\prime}>1$, we combine Eqs. (12) and (24)-(28) and get

$$
\begin{gathered}
m_{x}^{(j)}=-\frac{\eta b}{p_{o p t}-\eta} n_{x}^{(j)}, \quad m_{y}^{(j)}=-\frac{\eta b}{p_{o p t}-\eta} n_{y}^{(j)}, \quad m_{z}=\frac{2 \beta-\eta b n_{z}}{p_{o p t}-\eta}, \\
c^{\prime} m_{x}^{\prime(j)}=-\frac{\eta^{\prime} b^{\prime}}{p_{o p t}-\eta^{\prime}} n_{x}^{(j)}, \quad c^{\prime} m_{y}^{\prime(j)}=-\frac{\eta^{\prime} b^{\prime}}{p_{o p t}-\eta^{\prime}} n_{y}^{\prime(j)}, \quad c^{\prime} m_{z}^{\prime}=\frac{2 \beta-\eta^{\prime} b^{\prime} n_{z}^{\prime}}{p_{o p t}-\eta^{\prime}} .
\end{gathered}
$$

We discuss various situations based on whether $m_{z}=0\left(m_{z}^{\prime}=0\right)$ or $m_{z} \neq 0\left(m_{z}^{\prime} \neq 0\right)$. As we will see, the optimal measurement operators of one of the sets may become zero.

1. $m_{z} \neq 0$ and $m_{z}^{\prime} \neq 0$ 
Let $m_{z} \neq 0$ and $m_{z}^{\prime} \neq 0$. Then, as mentioned above, the signs of $m_{z}$ and $m_{z}^{\prime}$ must be opposite. Let us, without loss of generality, assume that $m_{z}>0$ and $m_{z}^{\prime}<0$ which, by Eqs. (34) and (35), is equivalent to the condition

$$
\frac{\eta b n_{z}}{2}<\beta<\frac{\eta^{\prime} b^{\prime} n_{z}^{\prime}}{2}
$$

In this case, the points of the Bloch sphere representing the optimal measurement operators of the first and second sets are in upper and lower hemispheres, respectively. From this consideration and the fact that POM elements must hold a resolution of the identity, it follows that neither the optimal POM elements associated to the first nor to the second set are zero at a whole. Therefore, due to the orthogonality of any optimal measurement operator and its corresponding conjugate state, conjugate states of any set cannot be full rank and hence must be pure i.e. $c=c^{\prime}=1$.

To obtain $p_{\text {opt }}$, first we find $\beta$ by using Eqs. (34) and (35) in

$$
\begin{aligned}
& \left(m_{x}^{(1)}\right)^{2}+\left(m_{y}^{(1)}\right)^{2}+\left(m_{z}\right)^{2}=1, \\
& \left(m_{x}^{\prime(1)}\right)^{2}+\left(m_{y}^{\prime(1)}\right)^{2}+\left(m_{z}^{\prime}\right)^{2}=1
\end{aligned}
$$

and subtracting the resulted equations. The result is

$$
\beta=\frac{\eta^{2}\left(b^{2}-1\right)-\eta^{\prime 2}\left(b^{\prime 2}-1\right)+2\left(\eta-\eta^{\prime}\right) p_{\text {opt }}}{4\left(\eta b n_{z}-\eta^{\prime} b^{\prime} n_{z}^{\prime}\right)},
$$

Then placing $\beta$ in the equation resulted from the first equation of (37), gives the desired result as

$$
p_{\text {opt }}=\frac{-B \pm \sqrt{B^{2}-A C}}{2 A}
$$

where

$$
\begin{aligned}
A= & \left(\eta-\eta^{\prime}\right)^{2}-\left(\eta b n_{z}-\eta^{\prime} b^{\prime} n_{z}^{\prime}\right)^{2}, \\
B= & {\left[\eta^{2}\left(b^{2}-1\right)-\eta^{\prime 2}\left(b^{\prime 2}-1\right)\right]\left(\eta-\eta^{\prime}\right) } \\
& -2 \eta b n_{z}\left(\eta-\eta^{\prime}\right)\left(\eta b n_{z}-\eta^{\prime} b^{\prime} n_{z}^{\prime}\right)+2 \eta\left(\eta b n_{z}-\eta^{\prime} b^{\prime} n_{z}^{\prime}\right)^{2}, \\
C= & {\left[\eta^{2}\left(b^{2}-1\right)-\eta^{\prime 2}\left(b^{\prime 2}-1\right)\right]^{2}+4 \eta^{2}\left(b^{2}-1\right)\left(\eta b n_{z}-\eta^{\prime} b^{\prime} n_{z}^{\prime}\right)^{2} } \\
& \quad-4 \eta b n_{z}\left[\eta^{2}\left(b^{2}-1\right)-\eta^{\prime 2}\left(b^{\prime 2}-1\right)\right]\left(\eta b n_{z}-\eta^{\prime} b^{\prime} n_{z}^{\prime}\right) .
\end{aligned}
$$

Of course, from two roots of Eq. (39), we must take the biggest one as $p_{\text {opt }}$.

2. $m_{z}=0$ and $m_{z}^{\prime}=0$

When $m_{z}=m_{z}^{\prime}=0$, from Eqs. (34) and (35) we have

$$
\frac{\eta b n_{z}}{2}=\beta=\frac{\eta^{\prime} b^{\prime} n_{z}^{\prime}}{2} .
$$

Here, the points representing optimal measurement operators are all on equator of the Bloch Sphere. In this case, the success probability is given by

$$
p_{\text {opt }}=\eta\left(1+b \sqrt{1-n_{z}^{2}}\right)
$$


and Eqs. (34) and (35) give $c^{\prime}$ as

$$
c^{\prime}=\frac{\eta^{\prime} b^{\prime}}{p_{\text {opt }}-\eta^{\prime}} \sqrt{1-n_{z}^{2}}
$$

In the case of $c^{\prime}<1$, all of $\lambda_{j}^{\prime}$ 's and hence primed measurement operators have to be zero while in the case of $c^{\prime}=1$ it is not necessarily the case.

3. $m_{z}=0$ and $m_{z}^{\prime} \neq 0$

In this case, Eqs. (34) and (35) lead to

$$
\frac{\eta b n_{z}}{2}=\beta \neq \frac{\eta^{\prime} b^{\prime} n_{z}^{\prime}}{2}
$$

For $n>1$, when this occurs, the success probability becomes as

$$
p_{\text {opt }}=\eta\left(1+b \sqrt{1-n_{z}^{2}}\right)
$$

and

$$
c^{\prime}=\frac{\sqrt{\eta^{2} b^{2} n_{z}^{2}+\eta^{\prime 2} b^{2}-2 \eta \eta^{\prime} b b^{\prime} n_{z} n_{z}^{\prime}}}{p_{\text {opt }}-\eta^{\prime}} .
$$

Whether primed conjugate states are mixed, $c^{\prime}<1$, or pure, $c^{\prime}=1$, the coefficients $\lambda_{j}^{\prime}$ 's and hence $\Pi_{j}^{\prime}$ 's should all be zero, since otherwise the measurement operators cannot satisfy a resolution of the identity. Here, the optimal measurement is the one which optimally distinguishes the states $\rho_{1}, \ldots, \rho_{n}$ only. Furthermore, Eq. (33) by using Eq. (34) imposes the constraint

$$
\sum_{j=1}^{n} \lambda_{j}\left(n_{x}^{(j)} \mathbf{i}+n_{y}^{(j)} \mathbf{j}\right)=0,
$$

which means that sates of the first set do not all place on the same half of the equator of the Bloch sphere.

In the case $n=1, n^{\prime}>1$, in order to satisfy the conditions (17) and (88), we have $\lambda_{j}^{\prime}=0$ for all $j$, and

$$
p_{\text {opt }}=\eta, \quad \Pi_{1}=\mathrm{I} .
$$

4. $m_{z} \neq 0$ and $m_{z}^{\prime}=0$

In this case, we have $c=c^{\prime}=1$ and

$$
\frac{\eta b n_{z}}{2} \neq \beta=\frac{\eta^{\prime} b^{\prime} n_{z}^{\prime}}{2}
$$

Also, for $n^{\prime}>1$, we obtain

$$
\begin{gathered}
p_{\text {opt }}=\eta^{\prime}\left(1+b^{\prime} \sqrt{1-n_{z}^{\prime 2}}\right) \\
\sum_{j=1}^{n^{\prime}} \lambda_{j}^{\prime}\left(n_{x}^{\prime(j)} \mathbf{i}+n_{y}^{\prime(j)} \mathbf{j}\right)=0
\end{gathered}
$$


and for $n>1$ and $n^{\prime}=1$, we have

$$
p_{\text {opt }}=\eta^{\prime}, \quad \Pi_{1}^{\prime}=\mathrm{I}
$$

For the case $c \leq 1$ and $c^{\prime}=1$, the roles of parameters for the first and second set of states are reversed and the results are the same as the case $c=1$ and $c^{\prime} \leq 1$ only with unprimed and primed parameters exchanged.

\section{Some special cases}

In this section, we derive analytical expressions for the maximal success probability and optimal detection operators in some instances which extend and confirm the results obtained in Refs. [19]-[21]. Hereafter, we use the notations

$$
\vec{\Lambda}^{(n)}=\sum_{j=1}^{n} \lambda_{j}\left(n_{x}^{(j)} \mathbf{i}+n_{y}^{(j)} \mathbf{j}\right), \quad \vec{\Lambda}^{\prime\left(n^{\prime}\right)}=\sum_{j=1}^{n^{\prime}} \lambda_{j}^{\prime}\left(n_{x}^{\prime(j)} \mathbf{i}+n_{y}^{\prime(j)} \mathbf{j}\right)
$$

\section{Case 1.}

Consider $n+1$ different pure states with the Bloch vectors

$$
\hat{n}_{j}=\left(n_{x}^{(j)}, n_{y}^{(j)}, n_{z}\right), \quad \hat{n}_{1}^{\prime}=(0,0,1), \quad j=1, \ldots, n .
$$

From Eqs. (33) and (35) we see that $m_{x}^{\prime(1)}=m_{y}^{\prime(1)}=0$ and $\vec{\Lambda}^{(n)}=0$. Some simple algebra, gives

$$
p_{\text {opt }}=\left\{\begin{array}{ccc}
\frac{2 \eta^{\prime}\left(\eta n_{z}-\eta^{\prime}\right)}{\eta\left(1+n_{z}\right)-2 \eta^{\prime}} & \text { if } \quad \eta n_{z}<\frac{2 \eta^{\prime}\left(\eta-\eta^{\prime}\right)}{\eta\left(1+n_{z}\right)-2 \eta^{\prime}}<\eta^{\prime} \quad \text { or } \quad \eta n_{z}>\frac{2 \eta^{\prime}\left(\eta-\eta^{\prime}\right)}{\eta\left(1+n_{z}\right)-2 \eta^{\prime}}>\eta^{\prime}, \\
& \text { and } \quad \frac{\eta}{p_{\text {opt }}-\eta} \vec{\Lambda}^{(n)}+\frac{\eta^{\prime}}{p_{\text {opt }}-\eta^{\prime}} \lambda_{1}^{\prime} \mathbf{j}=0 ; \\
\eta\left(1+\sqrt{1-n_{z}^{2}}\right) & \text { if } \quad \eta n_{z}=\eta^{\prime} \quad \text { or } \quad \eta \leq \frac{n_{z}-1-\sqrt{1-n_{z}^{2}}}{n_{z}^{2}+\eta n_{z}-1-n-(1+n) \sqrt{1-n_{z}^{2}}}, \quad \vec{\Lambda}^{(n)}=0 .
\end{array}\right.
$$

These results are reached by the following sets of detection operators, respectively

$$
\begin{aligned}
\Pi_{j} & =\lambda_{j}\left[I+\frac{\eta}{p_{o p t}-\eta}\left(n_{x}^{(j)} \sigma_{x}+n_{y}^{(j)} \sigma_{y}\right)-\frac{\left(\eta-\eta^{\prime}\right) p_{o p t}-\eta n_{z}\left(\eta n_{z}-\eta^{\prime}\right)}{\left(\eta n_{z}-\eta^{\prime}\right)\left(p_{o p t}-\eta\right)} \sigma_{z}\right], \\
\Pi_{1}^{\prime} & =\lambda_{1}^{\prime}\left[I-\frac{\left(\eta-\eta^{\prime}\right) p_{o p t}-\eta^{\prime}\left(\eta n_{z}-\eta^{\prime}\right)}{\left(\eta n_{z}-\eta^{\prime}\right)\left(p_{o p t}-\eta^{\prime}\right)} \sigma_{z}\right],
\end{aligned}
$$

and

$$
\begin{aligned}
\Pi_{j} & =\lambda_{j}\left(I+\frac{n_{x}^{(j)}}{\sqrt{1-n_{z}^{2}}} \sigma_{x}+\frac{n_{y}^{(j)}}{\sqrt{1-n_{z}^{2}}} \sigma_{y}\right) \\
\Pi_{1}^{\prime} & =0 .
\end{aligned}
$$

Case 2.

Consider $n+1$ different pure states with the Bloch vectors

$$
\hat{n}_{j}=\left(n_{x}^{(j)}, n_{y}^{(j)}, n_{z}\right), \quad \hat{n}_{1}^{\prime}=(0,1,0), \quad j=1, \ldots, n .
$$


Here, we obtain

$$
p_{\text {opt }}= \begin{cases}\frac{2 \eta^{2} \eta^{\prime} n_{z}^{2}}{\eta^{2} n_{z}^{2}-\left(\eta-\eta^{\prime}\right)^{2}} & \text { if } \quad 0<\frac{\left(\eta-\eta^{\prime}\right) \eta^{\prime}}{\left(\eta n_{z}\right)^{2}-\left(\eta-\eta^{\prime}\right)^{2}}<\frac{1}{2}, \quad \frac{\eta}{p_{o p t}-\eta} \vec{\Lambda}^{(n)}+\frac{\eta^{\prime}}{p_{\text {opt }}-\eta^{\prime}} \lambda_{1}^{\prime} \mathbf{j}=0 \\ 2 \eta & \text { if } \quad n_{z}=0, \quad \eta \geq \frac{1}{1+n}, \quad \vec{\Lambda}^{(n)}+\lambda_{1}^{\prime} \mathbf{j}=0 \\ \eta\left(1+\sqrt{1-n_{z}^{2}}\right) & \text { if } \quad n_{z} \neq 0, \quad \eta \geq \frac{1}{n+\sqrt{1-n_{z}^{2}}}, \quad \vec{\Lambda}^{(n)}=0 \\ \eta & \text { if } \quad n_{z} \neq 0, \quad \eta=\frac{1}{1+n}\end{cases}
$$

where are reached by the following set of detection operators, respectively

$$
\begin{gathered}
\Pi_{j}=\lambda_{j}\left[I+\frac{\eta}{p_{o p t}-\eta}\left(n_{x}^{(j)} \sigma_{x}+n_{y}^{(j)} \sigma_{y}\right)-\frac{\left(\eta-\eta^{\prime}\right) p_{o p t}-\eta^{2} n_{z}^{2}}{\eta n_{z}\left(p_{o p t}-\eta\right)} \sigma_{z}\right], \\
\Pi_{1}^{\prime}=\lambda_{1}^{\prime}\left[I+\frac{\eta^{\prime}}{p_{o p t}-\eta^{\prime}} \sigma_{y}-\frac{\left(\eta-\eta^{\prime}\right) p_{o p t}}{\eta n_{z}\left(p_{o p t}-\eta^{\prime}\right)} \sigma_{z}\right] ; \\
\Pi_{j}=\lambda_{j}\left(I+n_{x}^{(j)} \sigma_{x}+n_{y}^{(j)} \sigma_{y}\right), \\
\Pi_{1}^{\prime}=\lambda_{1}^{\prime}\left(I+\sigma_{y}\right) ; \\
\Pi_{j}=\lambda_{j}\left[I+\frac{1}{\sqrt{1-n_{z}^{2}}}\left(n_{x}^{(j)} \sigma_{x}+n_{y}^{(j)} \sigma_{y}\right)\right], \\
\Pi_{1}^{\prime}=0 ;
\end{gathered}
$$

and

$$
\begin{aligned}
& \Pi_{j}=0, \\
& \Pi_{1}^{\prime}=I .
\end{aligned}
$$

\section{Case 3.}

As a final case, let us consider two set such that each one containing two pure states with Bloch vectors as follows

$$
\hat{n}_{j}=\left(n_{x}^{(j)}, n_{y}^{(j)}, n_{z}\right), \quad \hat{n}_{j}^{\prime}=\left(n_{x}^{\prime(j)}, n_{y}^{\prime(j)}, 0\right), \quad j=1,2 .
$$

By referring to the general case discussed above, the optimal success probability and measurement have given by

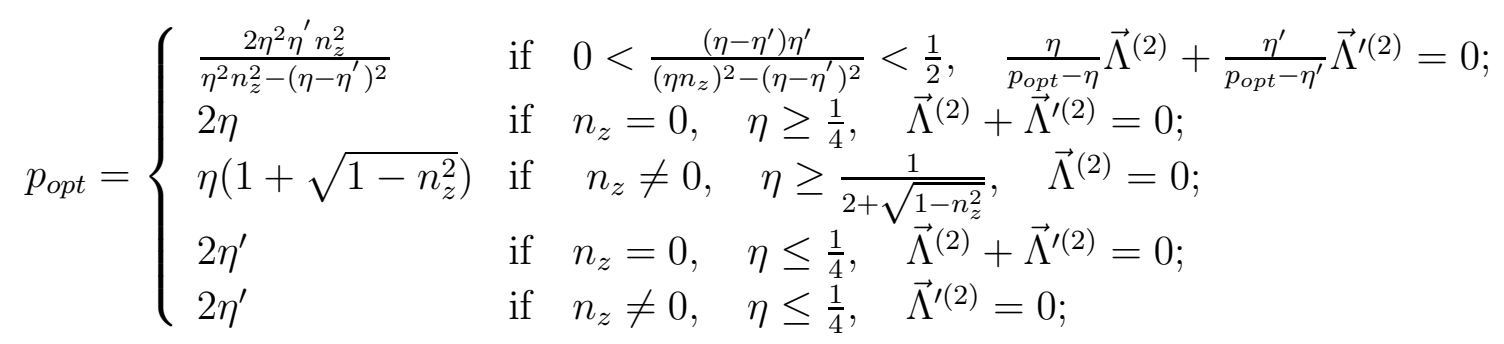

and the associated measurement operators are respectively given by

$$
\begin{gathered}
\Pi_{j}=\lambda_{j}\left[I+\frac{\eta}{p_{o p t}-\eta}\left(n_{x}^{(j)} \sigma_{x}+n_{y}^{(j)} \sigma_{y}\right)-\frac{\left(\eta-\eta^{\prime}\right) p_{o p t}-\eta^{2} n_{z}^{2}}{\eta n_{z}\left(p_{o p t}-\eta\right)} \sigma_{z}\right] \\
\Pi_{j}^{\prime}=\lambda_{j}^{\prime}\left[I+\frac{\eta^{\prime}}{p_{o p t}-\eta^{\prime}}\left(n_{x}^{\prime(j)} \sigma_{x}+n_{y}^{\prime(j)} \sigma_{y}\right)-\frac{\left(\eta-\eta^{\prime}\right) p_{o p t}}{\eta n_{z}\left(p_{o p t}-\eta^{\prime}\right)} \sigma_{z}\right] \\
\Pi_{j}=\lambda_{j}\left[I+\frac{1}{2}\left(n_{x}^{(j)} \sigma_{x}+n_{y}^{(j)} \sigma_{y}\right)\right] \\
\Pi_{j}^{\prime}=\lambda_{j}^{\prime}\left(I+n_{x}^{\prime(j)} \sigma_{x}+n_{y}^{\prime(j)} \sigma_{y}\right)
\end{gathered}
$$




$$
\begin{gathered}
\Pi_{j}=\lambda_{j}\left[I+\frac{1}{\sqrt{1-n_{z}^{2}}}\left(n_{x}^{(j)} \sigma_{x}+n_{y}^{(j)} \sigma_{y}\right)\right], \\
\Pi_{j}^{\prime}=0 ; \\
\quad \Pi_{j}=\lambda_{j}\left(I+n_{x}^{(j)} \sigma_{x}+n_{y}^{(j)} \sigma_{y}\right), \\
\Pi_{j}^{\prime}=\lambda_{j}^{\prime}\left(I+n_{x}^{\prime(j)} \sigma_{x}+n_{y}^{\prime(j)} \sigma_{y}\right) ;
\end{gathered}
$$

and

$$
\begin{aligned}
& \Pi_{j}=0, \\
& \Pi_{j}^{\prime}=\lambda_{j}^{\prime}\left(I+n_{x}^{(j)} \sigma_{x}+n_{y}^{\prime(j)} \sigma_{y}\right) .
\end{aligned}
$$

\section{MED between two sets of similarity transformed $m$-qubit states in general- ized Bloch sphere}

We consider particular $m$-qubit states in $d=2^{m}$ dimensional Hilbert space which possess properties similar to qubit density matrices represented in Bloch sphere, and so we call them generalized Bloch sphere states. Then the decomposition of these density matrices into a Bloch vector has, in general, the following form:

$$
\rho=\frac{1}{2^{m}}\left(I+a \sum_{i=1}^{2 m+1} n_{i} \gamma_{i}\right)=\frac{1}{2^{m}}(I+a \hat{n} \cdot \vec{\gamma}),
$$

where $\gamma_{i}$ for $i=1,2, \ldots, 2 m+1$, known as Dirac matrices, are generators of special orthogonal group $S O(2 m+1)$, and represented as traceless Hermitian matrices in a $2^{m}$-dimensional Hilbert space. That is, $\gamma_{i}$ are maximally anticommuting set which satisfy

$$
\left\{\gamma_{i}, \gamma_{j}\right\}=2 \delta_{i j} I_{d}
$$

For a brief review about Dirac matrices and an explicit construction of $\gamma_{i} \mathrm{~s}$, we refer the reader to [22] or the Appendix $A$ of [23]. From the properties (72), it is easy to see that $(\hat{n} \cdot \vec{\gamma})^{2}=I$ and so the eigenvalues of $\rho$ are $\frac{1 \pm a}{2^{m}}$. Therefore, the spectral decomposed form of the density matrix $\rho$ is

$$
\rho=\frac{1+a}{2^{m}}\left(\frac{I+\hat{n} \cdot \vec{\gamma}}{2}\right)+\frac{1-a}{2^{m}}\left(\frac{I-\hat{n} \cdot \vec{\gamma}}{2}\right)
$$

where $\frac{I+\hat{n} \cdot \vec{\gamma}}{2}$ and $\frac{I-\hat{n} \cdot \vec{\gamma}}{2}$ are projection operators (idempotents) to the degenerate eigenspaces corresponding to the eigenvalues $\frac{1+a}{2^{m}}$ and $\frac{1-a}{2^{m}}$, respectively. Here, $U_{i}=e^{i \sum_{i<j} \theta_{i j} \gamma_{i} \gamma_{j}}$ are spinor representations of the group $S O(2 m+1)$. It should be noticed that $U_{i}$ 's can also be chosen as spinor representation of any subgroup of $S O(2 m+1)$ of the same rank $m$ (maximal subgroup) such as $S O\left(i_{1}\right) \otimes S O\left(i_{2}\right) \otimes \ldots \otimes S O\left(i_{l}\right)$, where $\left(i_{1}, i_{2}, \ldots, i_{l}\right)$ is an arbitrary partition of $2 m+1$ to $l$ parties, i.e., $2 m+1=i_{1}+i_{2}+\ldots+i_{l}$. We write $\rho_{j}$ as

$$
\rho_{j}=\frac{1}{2^{m}}\left(I+b \sum_{i \in S_{V}} n_{i}^{(j)} \gamma_{i}+b \sum_{i \in S_{I}} n_{i} \gamma_{i}\right),
$$

where $S_{V}$ and $S_{I}$ stand for index sets of Bloch vector components which are respectively variant and invariant under unitary similarity transformations by $U_{j}$ 's. All of the discussions about qubit states can be extended to this case. 


\section{A. irreducible case}

When $U_{i}^{\prime}$ 's and $U_{i}^{\prime}$ 's are irreducible representations, as in qubit case of subsection $2, \mathcal{M}$ is proportional to identity. Also, by Schur's lemma, invariant part of the density operators and their associated conjugate states are just equal to $\frac{1}{2^{m}} I$. As in the qubit case, at least one of $c$ and $c^{\prime}$ is equal to one and here we take $c=1$. Since the discussion of $c^{\prime}<1$ is similar to the qubit one, we proceed with $c^{\prime}=1$ only. Therefore, we have

$$
\begin{aligned}
\rho_{j} & =\frac{1}{2^{m}}\left(I+b \sum_{i \in S_{V}} n_{i}^{(j)} \gamma_{i}\right), \\
\rho_{j}^{\prime} & =\frac{1}{2^{m}}\left(I+b^{\prime} \sum_{i \in S_{V}^{\prime}} n_{i}^{\prime(j)} \gamma_{i}\right), \\
\tau_{j} & =\frac{1}{2^{m}}\left(I+\sum_{i \in S_{V}} m_{i}^{(j)} \gamma_{i}\right), \\
\tau_{j}^{\prime} & =\frac{1}{2^{m}}\left(I+\sum_{i \in S_{V}^{\prime}} m_{i}^{(j)} \gamma_{i}\right) .
\end{aligned}
$$

To satisfy Eq. (8), we must choose the optimal POM elements as

$$
\begin{aligned}
& \Pi_{j}=\lambda_{j} U_{j} \pi_{1} U_{j}^{-1}=\lambda_{j}\left(I-\sum_{i \in S_{V}} m_{i}^{(j)} \gamma_{i}\right), \\
& \Pi_{j}^{\prime}=\lambda_{j}^{\prime} U_{j} \pi_{1}^{\prime} U_{j}^{-1}=\lambda_{j}^{\prime}\left(I-\sum_{i \in S_{V}^{\prime}} m_{i}^{\prime(j)} \gamma_{i}\right) .
\end{aligned}
$$

By using Eqs. (12) and (75)-(78), the components of Bloch vectors associated with optimal measurement are found as

$$
m_{i}^{(j)}=-\frac{\eta b}{p-\eta} n_{i}^{(j)}, \quad \quad m_{i}^{\prime(j)}=-\frac{\eta^{\prime} b^{\prime}}{p-\eta^{\prime}} n_{i}^{(j)}, \quad i \in S_{V}
$$

Finally, from the fact that the vectors $\hat{m}^{(j)}, \hat{m}^{\prime(j)}, \hat{n}^{(j)}$ and $\hat{n}^{\prime(j)}$ have unit lengths, the minimum error probability is found

$$
p_{\text {opt }}=\eta(1+b)=\eta^{\prime}\left(1+b^{\prime}\right) .
$$

which is in agreement with the general result of irreducible case, i.e. Eq. (19), for $d=2^{m}$, $a_{\max }=\frac{1+b}{2^{m}}$ and $a_{\text {max }}^{\prime}=\frac{1+b^{\prime}}{2^{m}}$.

\section{B. reducible case}

Let $U_{j}$ 's and $U_{j}^{\prime}$ 's are reducible representations. Since the operators $U_{j}^{\prime}$ 's and $U_{j}^{\prime}$ 's commute 
with $\mathcal{M}$, the index set associated to the maximal number of $\gamma_{i}$ 's which remain invariant under similarity transformations produced by them is $S_{I} \cap S_{I}^{\prime}$. Thus we have

$$
\mathcal{M}=\alpha I_{d}+\sum_{i \in S_{I} \cap S_{I}^{\prime}} \beta_{i} \gamma_{i} .
$$

As any optimal POM element is perpendicular to the corresponding conjugate state, hence, for any $j$, we have

$$
\begin{aligned}
& \Pi_{j}=\lambda_{j} U_{j} \pi_{1} U_{j}^{-1}=\lambda_{j}\left(I-\sum_{i \in S_{V}} m_{i}^{(j)} \gamma_{i}-\sum_{i \in S_{I}} m_{i} \gamma_{i}\right), \\
& \Pi_{j}^{\prime}=\lambda_{j}^{\prime} U_{j} \pi_{1}^{\prime} U_{j}^{-1}=\lambda_{j}^{\prime}\left(I-\sum_{i \in S_{V}^{\prime}} m_{i}^{\prime(j)} \gamma_{i}-\sum_{i \in S_{I}^{\prime}} m_{i}^{\prime} \gamma_{i}\right) .
\end{aligned}
$$

Placing these relations into the completeness relation (7), we obtain

$$
\begin{gathered}
\sum_{j=1}^{n} \lambda_{j}+\sum_{j=1}^{n^{\prime}} \lambda_{j}^{\prime}=1 \\
\sum_{j=1}^{n} \lambda_{j} m_{i}^{(j)}+\sum_{j=1}^{n^{\prime}} \lambda_{j}^{\prime} m_{i}^{(j)}=0, \quad i \in S_{V} \cap S_{V}^{\prime} \\
m_{i} \sum_{j=1}^{n} \lambda_{j}+m_{i}^{\prime} \sum_{j=1}^{n^{\prime}} \lambda_{j}^{\prime}=0, \quad i \in S_{I} \cap S_{I}^{\prime} \\
\sum_{j=1}^{n} \lambda_{j} m_{i}^{(j)}+m_{i}^{\prime} \sum_{j=1}^{n^{\prime}} \lambda_{j}^{\prime}=0, \quad i \in S_{V}-\left(S_{V} \cap S_{V}^{\prime}\right) \\
m_{i} \sum_{j=1}^{n} \lambda_{j}+\sum_{j=1}^{n^{\prime}} \lambda_{j}^{\prime} m_{i}^{\prime(j)}=0, \quad i \in S_{V}^{\prime}-\left(S_{V} \cap S_{V}^{\prime}\right)
\end{gathered}
$$

By substituting $\mathcal{M}$ from Eq. (83) into Eq. (12), we conclude that

$$
\begin{aligned}
& m_{i}^{(j)}=-\frac{\eta b}{p_{o p t}-\eta} n_{i}^{(j)}, \quad i \in S_{V} ; \\
& m_{i}=-\frac{\eta b}{p_{o p t}-\eta} n_{i}, \quad i \in S_{V}-\left(S_{V} \cap S_{V}^{\prime}\right) ; \\
& m_{i}=\frac{2^{m} \beta_{i}-\eta b n_{i}}{p_{o p t}-\eta}, \quad i \in S_{I} \cap S_{I}^{\prime},
\end{aligned}
$$

and

$$
\begin{aligned}
& m_{i}^{\prime(j)}=-\frac{\eta^{\prime} b^{\prime}}{p_{o p t}-\eta^{\prime}} n_{i}^{\prime(j)}, \quad i \in S_{V}^{\prime} ; \\
& m_{i}^{\prime}=-\frac{\eta^{\prime} b^{\prime}}{p_{o p t}-\eta^{\prime}} n_{i}^{\prime}, \quad i \in S_{V}^{\prime}-\left(S_{V} \cap S_{V}^{\prime}\right) ; \\
& m_{i}^{\prime}=\frac{2^{m} \beta_{i}-\eta^{\prime} b^{\prime} n_{i}^{\prime}}{p_{o p t}-\eta^{\prime}}, \quad i \in S_{I} \cap S_{I}^{\prime} .
\end{aligned}
$$


To derive $p_{\text {opt }}$ by solving Eqs. (88), (91) and (92), first we denote restrictions of $\hat{n}$ and $\hat{n}^{\prime}$ to the subspace corresponding to the index set $S_{I} \cap S_{I}^{\prime}$ by $\vec{n}_{0}$ and $\vec{n}_{0}^{\prime}$ respectively. Next, to simplify the algebra, we choose a coordinate system in the subspace corresponding to the index set $S_{I} \cap S_{I}^{\prime}$ such that the vector $\vec{n}_{0}$ is directed along an axis. In this coordinate system, let us denote by $n_{0}^{\prime}$ the component of $\vec{n}_{0}^{\prime}$ along $\vec{n}_{0}$ and by $n_{1}^{\prime}$ its component along an axis in the plane of $\vec{n}_{0}$ and $\vec{n}_{0}^{\prime}$ which is perpendicular to $\vec{n}_{0}$. By some tricky algebra which is discussed in detail in Appendix C, we find

$$
p_{\text {opt }}=\frac{-B \pm \sqrt{B^{2}-A C}}{2 A},
$$

where

$$
\begin{aligned}
A= & \left(\eta-\eta^{\prime}\right)^{2}-\left(\eta b n_{0}-\eta^{\prime} b^{\prime} n_{0}^{\prime}\right)^{2}-\eta^{\prime 2} b^{\prime 2} n_{1}^{\prime 2} \\
B= & {\left[\eta^{2}\left(1-b^{2}\right)-\eta^{\prime 2}\left(1-b^{\prime 2}\right)-\frac{2 \eta \eta^{\prime 2} b b^{\prime 2} n_{0} n_{1}^{\prime 2}}{\eta b n_{0}-\eta^{\prime} b^{\prime} n_{0}^{\prime}}\right]\left(\eta-\eta^{\prime}\right) } \\
& -2 \eta b n_{0}\left(\eta-\eta^{\prime}\right)\left(\eta b n_{0}-\eta^{\prime} b^{\prime} n_{0}^{\prime}+\frac{\eta^{\prime 2} b^{\prime 2} n_{1}^{\prime 2}}{\eta b n_{0}-\eta^{\prime} b^{\prime} n_{0}^{\prime}}\right)+2 \eta\left[\left(\eta b n_{0}-\eta^{\prime} b^{\prime} n_{0}^{\prime}\right)^{2}+\eta^{\prime 2} b^{\prime 2} n_{1}^{\prime 2}\right] \\
C= & {\left[\eta^{2}\left(1-b^{2}\right)-\eta^{\prime 2}\left(1-b^{\prime 2}\right)-\frac{\eta \eta^{\prime 2} b b^{\prime 2} n_{0} n_{1}^{\prime 2}}{\eta b n_{0}-\eta^{\prime} b^{\prime} n_{0}^{\prime}}\right]^{2} } \\
& +4 \eta^{2}\left[b^{2}\left(1+\frac{\eta^{\prime 2} b^{\prime 2} n_{0}^{2} n_{1}^{\prime 2}}{\left(\eta b n_{0}-\eta^{\prime} b^{\prime} n_{0}^{\prime}\right)^{2}}\right)-1\right]\left[\left(\eta b n_{0}-\eta^{\prime} b^{\prime} n_{0}^{\prime}\right)^{2}+\eta^{\prime 2} b^{\prime 2} n_{1}^{\prime 2}\right] \\
& -4 \eta b n_{0}\left[\eta^{2}\left(1-b^{2}\right)-\eta^{\prime 2}\left(1-b^{\prime 2}\right)-\frac{2 \eta \eta^{\prime 2} b b^{\prime 2} n_{0} n_{1}^{\prime 2}}{\eta b n_{0}-\eta^{\prime} b^{\prime} n_{0}^{\prime}}\right]\left(\eta b n_{0}-\eta^{\prime} b^{\prime} n_{0}^{\prime}+\frac{\eta^{\prime 2} b^{\prime 2} n_{1}^{\prime 2}}{\eta b n_{0}-\eta^{\prime} b^{\prime} n_{0}^{\prime}}\right) .
\end{aligned}
$$

It is easy to see that the statement of $p_{\text {opt }}$ is reduced to the statement of qubit case if we let $S_{I}=S_{I}^{\prime}$ be one-dimensional and so $n_{1}^{\prime}=0, n_{0}=n_{z}$ and $n_{0}^{\prime}=n_{z}^{\prime}$.

\section{Conclusion}

Using the necessary and sufficient conditions for minimum-error discrimination in the equality form which is equivalent to Helstrom family of ensembles, we investigated minimum-error discrimination among two sets of different equiprobable quantum states where each set generated from a density operator by unitary similarity transformations. In the case that the unitary operators involved in the similarity transformations are generating of irreducible representations of two subgroups of $U(d)$, we precisely derived the maximum success probability and the optimal measurement. However, for the case that the representations are reducible, we did not solve optimality conditions in general and instead we illustrated the method by applying it to solve optimality conditions for two set of equiprobable qubit states together with some particular cases and two set of equiprobable m-qubit states. The presented particular cases were extended forms of some examples given in the literature and our results confirm theirs. 


\section{Appendix A}

Here, we want to calculate maximal success probability given in Eq. (93). To this aim, first we express Eq. (12) in terms of the Bloch vectors components as

$$
\begin{aligned}
\eta b n_{i}-\eta^{\prime} b^{\prime} n_{i}^{\prime} & =\left(p_{o p t}-\eta^{\prime}\right) m_{i}^{\prime}-\left(p_{o p t}-\eta\right) m_{i} \\
2^{m} \beta_{i} & =\eta b n_{i}+\left(p_{o p t}-\eta\right) m_{i}
\end{aligned}
$$

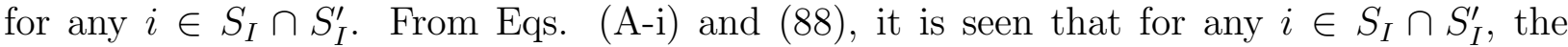
signs of $\eta b n_{i}-\eta^{\prime} b^{\prime} n_{i}^{\prime}$ and $m_{i}$ are opposite. In other words, if restrictions of $\hat{n}, \hat{n}^{\prime}, \hat{m}$ and $\hat{m}^{\prime}$ to the subspace corresponding to the index set $S_{I} \cap S_{I}^{\prime}$ are denoted by $\vec{n}_{0}, \vec{n}_{0}^{\prime}, \vec{m}_{0}$ and $\vec{m}_{0}^{\prime}$ respectively, then the vectors $\eta b \vec{n}_{0}-\eta^{\prime} b^{\prime} \vec{n}_{0}^{\prime}$ and $\vec{m}_{0}$ point in the opposite directions and we can write

$$
\vec{m}_{0}=-\sqrt{\sum_{i \in S_{I} \cap S_{I}^{\prime}}\left(m_{i}\right)^{2}} \frac{\eta b \vec{n}_{0}-\eta^{\prime} b^{\prime} \vec{n}_{0}^{\prime}}{\left|\eta b \vec{n}_{0}-\eta^{\prime} b^{\prime} \vec{n}_{0}^{\prime}\right|} .
$$

Therefore, Eq. (A-ii) takes the vectorial form

$$
2^{m} \vec{\beta}=\eta b \vec{n}_{0}-\left(p_{\text {opt }}-\eta\right) \sqrt{\sum_{i \in S_{I} \cap S_{I}^{\prime}}\left(m_{i}\right)^{2}} \frac{\eta b \vec{n}_{0}-\eta^{\prime} b^{\prime} \vec{n}_{0}^{\prime}}{\left|\eta b \vec{n}_{0}-\eta^{\prime} b^{\prime} \vec{n}_{0}^{\prime}\right|}
$$

Eqs. (88), (A-iii) and (A-iv) show that the vectors $\vec{n}_{0}, \vec{n}_{0}^{\prime}, \vec{\beta}, \vec{m}_{0}$ and $\vec{m}_{0}^{\prime}$ are coplanar. Next, to simplify the algebra, we choose an orthogonal coordinate system in the subspace corresponding to the index set $S_{I} \cap S_{I}^{\prime}$ such the plane of $\vec{n}_{0}$ and $\vec{n}_{0}^{\prime}$ coincide with the plane defined by an arbitrary pair of coordinate axes and $\vec{n}_{0}$ points to the positive direction of one axis of the pair. Let us denote by $n_{0}^{\prime}$ and $\beta_{0}$ the components of $\vec{n}_{0}^{\prime}$ and $\vec{\beta}$ along an axis of the pair lying in the direction of $\vec{n}_{0}$ and by $n_{1}^{\prime}$ and $\beta_{1}$ their components along another axis, respectively. In the considered frame, Eq. (88) and the third relations of Eqs. (91) and (92) are written as

$$
\begin{gathered}
\mu m_{0}+(1-\mu) m_{0}^{\prime}=0 \\
\mu m_{1}+(1-\mu) m_{1}^{\prime}=0 \\
m_{0}=\frac{2^{m} \beta_{0}-\eta b n_{0}}{p_{o p t}-\eta}, \\
m_{1}=\frac{2^{m} \beta_{1}}{p_{o p t}-\eta}, \\
m_{0}^{\prime}=\frac{2^{m} \beta_{0}-\eta^{\prime} b^{\prime} n_{0}^{\prime}}{p_{o p t}-\eta^{\prime}}, \\
m_{1}^{\prime}=\frac{2^{m} \beta_{1}-\eta^{\prime} b^{\prime} n_{1}^{\prime}}{p_{o p t}-\eta^{\prime}},
\end{gathered}
$$

where we have introduced $\mu=\sum_{j=1}^{n} \lambda_{j}$ and $m_{0}\left(m_{0}^{\prime}\right)$ and $m_{1}\left(m_{1}^{\prime}\right)$ are components of $\vec{m}_{0}$ $\left(\vec{m}_{0}^{\prime}\right)$. When we square both sides of the first two relations of Eqs. (91) and (92), then sum up over $i$ and use the unity of $\hat{n}, \hat{n}^{\prime}, \hat{m}$ and $\hat{m}^{\prime}$, we obtain

$$
\begin{gathered}
1-m_{0}^{2}-m_{1}^{2}=\frac{\eta^{2} b^{2}}{\left(p_{o p t}-\eta\right)^{2}}\left(1-n_{0}^{2}\right) \\
1-m_{0}^{\prime 2}-m_{1}^{\prime 2}=\frac{\eta^{\prime 2} b^{\prime 2}}{\left(p_{o p t}-\eta^{\prime}\right)^{2}}\left(1-n_{0}^{\prime 2}-n_{1}^{\prime 2}\right)
\end{gathered}
$$

Finally, by composing Eqs. (A-v)-( $\mathrm{A}-\mathrm{vii})$ we attain to Eq. (93) for $p_{\text {opt }}$. 


\section{References}

[1] C. W. Helstrom, Quantum Detection and Estimation theory, New York: Academic, (1976).

[2] A. Holevo, Probabilistic and Statistical Aspects of Quantum Theory, Amsterdam: North-Holland, (1982).

[3] Y. C. Eldar, A. Megretski, and G. C. Verghese, IEEE Trans. Inform. Theory 49, 1007 (2003).

[4] S. M. Barnett and S. Croke, J. Phys. A: Math. Theor. 42, 062001, (2009).

[5] S. M. Barnett and S. Croke, Adv. Opt. Photon. 1, 238, (2009).

[6] M.A. Jafarizadeh, Y. Mazhari, M. Aali, Quantum Inf Process, 10, 155 (2011).

[7] Won-Young Hwang and Joonwoo Bae, J. Math. Phys. 51, 022202 (2010).

[8] A. Assalini, G. Cariolaro and G. Pierobon, 81, 012315 (2010).

[9] I. D. Ivanovic, Phys. Lett. A 123, 257 (1987).

[10] D. Dieks, Phys. Lett. A 126, 303 (1988).

[11] A. Peres and D. R. Terno, J. Phys. A 31, 7105 (1998).

[12] G. Jaeger and A. Shimony, Phys. Lett. A 197, 83 (1995).

[13] A. Chefles, Phys. Lett. A 239, 339 (1998).

[14] M. A. Jafarizadeh, M. Rezaei, N. Karimi and A. R. Amiri, Phys. Rev. A 77, 042314 (2008).

[15] G. Kimura, T. Miyadera, and H. Imai, Phys. Rev. A 79, 062306 (2009).

[16] M. A. Jafarizadeh, R. Sufiani and Y. Mazhari Khiavi, DOI: 10.1103/PhysRevA.00.002100 (2011).

[17] A. W. Joshi, Elements of group theory for physicists, New Age International (P) Limited, Publishers, (1997).

[18] G. James and M. W. Liebeck, Representations and characters of groups, Cambridge University Press (2001).

[19] E. Andersson, S.M. Barnett, C.R. Gilson, and K. Hunter, Phys. Rev. A 65, 052308 (2002).

[20] B.F. Samsonov, Minimum error discrimination problem for pure qubit states, Phys. Rev. A 80, 052305 (2009). 
[21] M. Ježek, J. Řeháček and J. Fiurášek, Phys. Rev. A 65, 060301 (2002).

[22] S. Weinberg, The quantum theory of fields I, Cambridge University Press, N.Y. (1995).

[23] M.A. Jafarizadeh, R. Sufiani, Phys. Rev. A 77, 012105 (2008). 